\title{
Refinements of the Heinz inequalities for matrices
}

\author{
Yanxiong Yan ${ }^{1,2}$, Yuming Feng ${ }^{3}$ and Guiyun Chen ${ }^{1 *}$
}

"Correspondence:

gychen1963@163.com

${ }^{1}$ School of Mathematics and

Statistics, Southwest University,

Chongqing, 400715, P.R. China

Full list of author information is

available at the end of the article

\begin{abstract}
This article aims to discuss Heinz inequalities involving unitarily invariant norms. We use a similar method to (Feng in J. Inequal. Appl. 2012:18, 2012; Wang in J. Inequal. Appl. 2013:424, 2013) and we get different refinements of the Heinz inequalities for matrices. Our results are better than some given in (Kittaneh in Integral Equ. Oper. Theory 68:519-527, 2010) and they are different from (Feng in J. Inequal. Appl. 2012:18, 2012; Wang in J. Inequal. Appl. 2013:424, 2013).
\end{abstract}

Keywords: convex function; Heinz inequality; Hermite-Hadamard inequality; unitarily invariant norm

\section{Introduction}

If $A, B, X$ are operators on a complex separable Hilbert space such that $A$ and $B$ are positive, then for every unitarily invariant norm $\|\cdot\| \|$, the function $f(v)=\left\|A^{v} X B^{1-v}+A^{1-v} X B^{v}\right\|$ is convex on the interval $[0,1]$, attains its minimum at $v=\frac{1}{2}$, and attains its maximum at $v=0$ and $v=1$. Moreover, $f(v)=f(1-v)$ for $0 \leq v \leq 1$. From [1] we know that for every unitarily invariant norm, we have the Heinz inequalities

$$
2\left\|A^{\frac{1}{2}} X B^{\frac{1}{2}}\right\| \leq\left\|A^{v} X B^{1-v}+A^{1-v} X B^{v}\right\| \leq\|A X+X B\| .
$$

In [2], Feng used the following inequalities to get refinements of (1):

$$
f\left(\frac{a+b}{2}\right) \leq \frac{1}{b-a} \int_{a}^{b} f(t) d t \leq \frac{1}{4}\left(f(a)+2 f\left(\frac{a+b}{2}\right)+f(b)\right) \leq \frac{f(a)+f(b)}{2}
$$

where $f$ is a real-valued function which is convex on the interval $[a, b]$. With a similar method, Wang [3] got some new refinements of (1).

In this paper, we use a similar method to [2,3] and we get different refinements of (1).

When we consider $\|T\| \|$, we are implicitly assuming that the operator $T$ belongs to the norm ideal associated with $\|\mid \cdot\|$. Our results are better than those in [4] and different from $[2,3]$.

\section{Main results}

From page 122 of [5], we know the following Hermite-Hadamard integral inequality for convex functions.

@2014 Yan et al.; licensee Springer. This is an Open Access article distributed under the terms of the Creative Commons Attribution License (http://creativecommons.org/licenses/by/2.0), which permits unrestricted use, distribution, and reproduction in any medium, provided the original work is properly cited. 
Lemma 1 (Hermite-Hadamard integral inequality) Let $f$ be a real-valued function which is convex on the interval $[a, b]$. Then

$$
f\left(\frac{a+b}{2}\right) \leq \frac{1}{b-a} \int_{a}^{b} f(t) d t \leq \frac{f(a)+f(b)}{2} .
$$

We will use the following lemma.

Lemma 2 Let $f$ be a real-valued function which is convex on the interval $[a, b]$. Then

$$
f\left(\frac{a+b}{2}\right) \leq \frac{1}{b-a} \int_{a}^{b} f(t) d t \leq \frac{1}{8}\left(3 f(a)+2 f\left(\frac{a+b}{2}\right)+3 f(b)\right) \leq \frac{f(a)+f(b)}{2} .
$$

Proof Using the previous lemma, we can easily verify the inequality

$$
\frac{1}{8}\left(3 f(a)+2 f\left(\frac{a+b}{2}\right)+3 f(b)\right) \leq \frac{f(a)+f(b)}{2} .
$$

Next, we will prove the following inequality:

$$
\frac{1}{b-a} \int_{a}^{b} f(t) d t \leq \frac{1}{8}\left(3 f(a)+2 f\left(\frac{a+b}{2}\right)+3 f(b)\right) .
$$

From the previous lemma, we have

$$
\begin{aligned}
\frac{1}{b-a} \int_{a}^{b} f(t) d t & =\frac{1}{b-a}\left(\int_{a}^{\frac{a+b}{2}} f(t) d t+\int_{\frac{a+b}{2}}^{b} f(t) d t\right) \\
& \leq \frac{1}{b-a}\left(\frac{f(a)+f\left(\frac{a+b}{2}\right)}{2} \cdot \frac{b-a}{2}+\frac{f\left(\frac{a+b}{2}\right)+f(b)}{2} \cdot \frac{b-a}{2}\right) \\
& =\frac{1}{4}\left(f(a)+2 f\left(\frac{a+b}{2}\right)+f(b)\right) \\
& =\frac{1}{8}\left(2 f(a)+4 f\left(\frac{a+b}{2}\right)+2 f(b)\right) \\
& \leq \frac{1}{8}\left(2 f(a)+2 f\left(\frac{a+b}{2}\right)+(f(a)+f(b))+2 f(b)\right) \\
& =\frac{1}{8}\left(3 f(a)+2 f\left(\frac{a+b}{2}\right)+3 f(b)\right) .
\end{aligned}
$$

Applying the previous lemma to the function $f(v)=\left\|A^{v} X B^{1-v}+A^{1-v} X B^{v}\right\|$ on the interval $[\mu, 1-\mu]$ when $0 \leq \mu \leq \frac{1}{2}$, and on the interval $[1-\mu, \mu]$ when $\frac{1}{2} \leq \mu \leq 1$, we obtain a refinement of the first inequality in (1).

Theorem 1 Let $A, B, X$ be operators such that $A, B$ are positive. Then for $0 \leq \mu \leq 1$ and for every unitarily invariant norm, we have

$$
\begin{aligned}
2\left\|A^{\frac{1}{2}} X B^{\frac{1}{2}}\right\| & \leq \frac{1}{|1-2 \mu|}\left|\int_{\mu}^{1-\mu}\left\|A^{v} X B^{1-v}+A^{1-v} X B^{v}\right\| d v\right| \\
& \leq \frac{1}{4}\left(3\left\|A^{\mu} X B^{1-\mu}+A^{1-\mu} X B^{\mu}\right\|+2\left\|A^{\frac{1}{2}} X B^{\frac{1}{2}}\right\|\right) \\
& \leq\left\|A^{\mu} X B^{1-\mu}+A^{1-\mu} X B^{\mu}\right\| .
\end{aligned}
$$


Proof First assume that $0 \leq \mu \leq \frac{1}{2}$. Then it follows by the previous lemma that

$$
\begin{aligned}
f\left(\frac{1-\mu+\mu}{2}\right) & \leq \frac{1}{1-2 \mu} \int_{\mu}^{1-\mu} f(t) d t \\
& \leq \frac{1}{8}\left(3 f(\mu)+2 f\left(\frac{1-\mu+\mu}{2}\right)+3 f(1-\mu)\right) \\
& \leq \frac{f(\mu)+f(1-\mu)}{2},
\end{aligned}
$$

and so

$$
\begin{aligned}
f\left(\frac{1}{2}\right) & \leq \frac{1}{1-2 \mu} \int_{\mu}^{1-\mu} f(t) d t \\
& \leq \frac{1}{4}\left(3 f(\mu)+f\left(\frac{1}{2}\right)\right) \\
& \leq f(\mu) .
\end{aligned}
$$

Thus,

$$
\begin{aligned}
2\left\|A^{\frac{1}{2}} X B^{\frac{1}{2}}\right\| & \leq \frac{1}{1-2 \mu} \int_{\mu}^{1-\mu}\left\|A^{v} X B^{1-\nu}+A^{1-\nu} X B^{\nu}\right\| d v \\
& \leq \frac{1}{4}\left(3\left\|A^{\mu} X B^{1-\mu}+A^{1-\mu} X B^{\mu}\right\|+2\left\|A^{\frac{1}{2}} X B^{\frac{1}{2}}\right\|\right) \\
& \leq\left\|A^{\mu} X B^{1-\mu}+A^{1-\mu} X B^{\mu}\right\| .
\end{aligned}
$$

Now, assume that $\frac{1}{2} \leq \mu \leq 1$. Then by applying (3) to $1-\mu$, it follows that

$$
\begin{aligned}
2\left\|A^{\frac{1}{2}} X B^{\frac{1}{2}}\right\| & \leq \frac{1}{2 \mu-1} \int_{1-\mu}^{\mu}\left\|A^{v} X B^{1-\nu}+A^{1-\nu} X B^{v}\right\| d v \\
& \leq \frac{1}{4}\left(3\left\|A^{\mu} X B^{1-\mu}+A^{1-\mu} X B^{\mu}\right\|+2\left\|A^{\frac{1}{2}} X B^{\frac{1}{2}}\right\|\right) \\
& \leq\left\|A^{\mu} X B^{1-\mu}+A^{1-\mu} X B^{\mu}\right\| .
\end{aligned}
$$

Since

$$
\begin{aligned}
& \lim _{\mu \rightarrow \frac{1}{2}} \frac{1}{|1-2 \mu|}\left|\int_{\mu}^{1-\mu}\left\|A^{v} X B^{1-v}+A^{1-\nu} X B^{v}\right\| d v\right| \\
& \quad=\lim _{\mu \rightarrow \frac{1}{2}} \frac{1}{4}\left(3\left\|A^{\mu} X B^{1-\mu}+A^{1-\mu} X B^{\mu}\right\|+2\left\|A^{\frac{1}{2}} X B^{\frac{1}{2}}\right\|\right) \\
& \quad=2\left\|A^{\frac{1}{2}} X B^{\frac{1}{2}}\right\|,
\end{aligned}
$$

the inequalities in (2) follow by combining (3) and (4).

Applying the previous lemma to the function $f(v)=\left\|A^{v} X B^{1-v}+A^{1-v} X B^{v}\right\|$ on the interval $\left[\mu, \frac{1}{2}\right]$ when $0 \leq \mu \leq \frac{1}{2}$, and on the interval $\left[\frac{1}{2}, \mu\right]$ when $\frac{1}{2} \leq \mu \leq 1$, we obtain the following. 
Theorem 2 Let $A, B, X$ be operators such that $A, B$ are positive. Then for $0 \leq \mu \leq 1$ and for every unitarily invariant norm, we have

$$
\begin{aligned}
& \left\|A^{\frac{2 \mu+1}{4}} X B^{\frac{3-2 \mu}{4}}+A^{\frac{3-2 \mu}{4}} X B^{\frac{2 \mu+1}{4}}\right\| \\
& \leq \frac{2}{|1-2 \mu|}\left|\int_{\mu}^{\frac{1}{2}}\left\|A^{v} X B^{1-v}+A^{1-\nu} X B^{v}\right\| d v\right| \\
& \leq \frac{1}{8}\left(3\left\|A^{\mu} X B^{1-\mu}+A^{1-\mu} X B^{\mu}\right\|+2\left\|A^{\frac{2 \mu+1}{4}} X B^{\frac{3-2 \mu}{4}}+A^{\frac{3-2 \mu}{4}} X B^{\frac{2 \mu+1}{4}}\right\|+6\left\|A^{\frac{1}{2}} X B^{\frac{1}{2}}\right\|\right) \\
& \quad \leq \frac{1}{2}\left(\left\|A^{\mu} X B^{1-\mu}+A^{1-\mu} X B^{\mu}\right\|+2\left\|A^{\frac{1}{2}} X B^{\frac{1}{2}}\right\|\right)
\end{aligned}
$$

The inequality (5) and the first inequality in (1) yield the following refinement of the first inequality in (1).

Corollary 1 Let $A, B, X$ be operators such that $A, B$ are positive. Then for $0 \leq \mu \leq 1$ and for every unitarily invariant norm, we have

$$
\begin{aligned}
& 2\left\|A^{\frac{1}{2}} X B^{\frac{1}{2}}\right\| \\
& \leq\left\|A^{\frac{2 \mu+1}{4}} X B^{\frac{3-2 \mu}{4}}+A^{\frac{3-2 \mu}{4}} X B^{\frac{2 \mu+1}{4}}\right\| \\
& \leq \frac{2}{|1-2 \mu|}\left|\int_{\mu}^{\frac{1}{2}}\left\|A^{v} X B^{1-v}+A^{1-v} X B^{v}\right\| d v\right| \\
& \quad \leq \frac{1}{8}\left(3\left\|A^{\mu} X B^{1-\mu}+A^{1-\mu} X B^{\mu}\right\|+2\left\|A^{\frac{2 \mu+1}{4}} X B^{\frac{3-2 \mu}{4}}+A^{\frac{3-2 \mu}{4}} X B^{\frac{2 \mu+1}{4}}\right\|+6\left\|A^{\frac{1}{2}} X B^{\frac{1}{2}}\right\|\right) \\
& \leq \frac{1}{2}\left(\left\|A^{\mu} X B^{1-\mu}+A^{1-\mu} X B^{\mu}\right\|+2\left\|A^{\frac{1}{2}} X B^{\frac{1}{2}}\right\|\right) \\
& \leq\left\|A^{\mu} X B^{1-\mu}+A^{1-\mu} X B^{\mu}\right\| .
\end{aligned}
$$

Applying the previous lemma to the function $f(v)=\left\|A^{v} X B^{1-v}+A^{1-v} X B^{v}\right\|$ on the interval $[0, \mu]$ when $0 \leq \mu \leq \frac{1}{2}$, and on the interval $[\mu, 1]$ when $\frac{1}{2} \leq \mu \leq 1$, we obtain the following theorem.

Theorem 3 Let $A, B, X$ be operators such that $A, B$ are positive. Then

(1) for $0 \leq \mu \leq \frac{1}{2}$ and for every unitarily norm,

$$
\begin{aligned}
& \left\|A^{\frac{\mu}{2}} X B^{1-\frac{\mu}{2}}+A^{1-\frac{\mu}{2}} X B^{\frac{\mu}{2}}\right\| \\
& \quad \leq \frac{1}{\mu} \int_{0}^{\mu}\left\|A^{v} X B^{1-v}+A^{1-v} X B^{v}\right\| d v \\
& \leq \frac{1}{8}\left(3\|A X+X B\|+2\left\|A^{\frac{\mu}{2}} X B^{1-\frac{\mu}{2}}+A^{1-\frac{\mu}{2}} X B^{\frac{\mu}{2}}\right\|+3\left\|A^{\mu} X B^{1-\mu}+A^{1-\mu} X B^{\mu}\right\|\right) \\
& \quad \leq \frac{1}{2}\left(\|A X+X B\|+\left\|A^{\mu} X B^{1-\mu}+A^{1-\mu} X B^{\mu}\right\|\right) ;
\end{aligned}
$$

(2) for $\frac{1}{2} \leq \mu \leq 1$ and for every unitarily norm,

$$
\begin{aligned}
& \left\|A^{\frac{1+\mu}{2}} X B^{\frac{1-\mu}{2}}+A^{\frac{1-\mu}{2}} X B^{\frac{1+\mu}{2}}\right\| \\
& \quad \leq \frac{1}{1-\mu} \int_{\mu}^{1}\left\|A^{v} X B^{1-v}+A^{1-v} X B^{v}\right\| d v
\end{aligned}
$$




$$
\begin{aligned}
& \leq \frac{1}{8}\left(3\|A X+X B\|+2\left\|A^{\frac{1+\mu}{2}} X B^{\frac{1-\mu}{2}}+A^{\frac{1-\mu}{2}} X B^{\frac{1+\mu}{2}}\right\|+3\left\|A^{\mu} X B^{1-\mu}+A^{1-\mu} X B^{\mu}\right\|\right) \\
& \leq \frac{1}{2}\left(\|A X+X B\|+\left\|A^{\mu} X B^{1-\mu}+A^{1-\mu} X B^{\mu}\right\|\right) .
\end{aligned}
$$

Since the function $f(v)=\left\|A^{v} X B^{1-v}+A^{1-v} X B^{v}\right\|$ is decreasing on the interval $\left[0, \frac{1}{2}\right]$ and increasing on the interval $\left[\frac{1}{2}, 1\right]$, and using the inequalities (7) and (8), we obtain the refinement of the second inequality in (1).

Corollary 2 Let $A, B, X$ be operators such that $A, B$ are positive. Then for $0 \leq \mu \leq 1$ and for every unitarily invariant norm, we have the following.

(1) For $0 \leq \mu \leq \frac{1}{2}$ and for every unitarily norm,

$$
\begin{aligned}
& \left\|A^{\mu} X B^{1-\mu}+A^{1-\mu} X B^{\mu}\right\| \\
& \leq\left\|A^{\frac{\mu}{2}} X B^{1-\frac{\mu}{2}}+A^{1-\frac{\mu}{2}} X B^{\frac{\mu}{2}}\right\| \\
& \leq \frac{1}{\mu} \int_{0}^{\mu}\left\|A^{v} X B^{1-v}+A^{1-\nu} X B^{\nu}\right\| d v \\
& \leq \frac{1}{8}\left(3\|A X+X B\|+2\left\|A^{\frac{\mu}{2}} X B^{1-\frac{\mu}{2}}+A^{1-\frac{\mu}{2}} X B^{\frac{\mu}{2}}\right\|+3\left\|A^{\mu} X B^{1-\mu}+A^{1-\mu} X B^{\mu}\right\|\right) \\
& \quad \leq \frac{1}{2}\left(\|A X+X B\|+\left\|A^{\mu} X B^{1-\mu}+A^{1-\mu} X B^{\mu}\right\|\right) \\
& \leq\|A X+X B\| .
\end{aligned}
$$

(2) For $\frac{1}{2} \leq \mu \leq 1$ and for every unitarily norm,

$$
\begin{aligned}
&\left\|A^{\mu} X B^{1-\mu}+A^{1-\mu} X B^{\mu}\right\| \\
& \leq\left\|A^{\frac{1+\mu}{2}} X B^{\frac{1-\mu}{2}}+A^{\frac{1-\mu}{2}} X B^{\frac{1+\mu}{2}}\right\| \\
& \leq \frac{1}{1-\mu} \int_{\mu}^{1}\left\|A^{v} X B^{1-v}+A^{1-v} X B^{v}\right\| d \nu \\
& \leq \frac{1}{8}\left(3\|A X+X B\|+2\left\|A^{\frac{1+\mu}{2}} X B^{\frac{1-\mu}{2}}+A^{\frac{1-\mu}{2}} X B^{\frac{1+\mu}{2}}\right\|+3\left\|A^{\mu} X B^{1-\mu}+A^{1-\mu} X B^{\mu}\right\|\right) \\
& \leq \frac{1}{2}\left(\|A X+X B\|+\left\|A^{\mu} X B^{1-\mu}+A^{1-\mu} X B^{\mu}\right\|\right) \\
& \leq\|A X+X B\| .
\end{aligned}
$$

It should be noticed that in the inequalities (7) to (10), we have

$$
\begin{aligned}
\lim _{\mu \rightarrow 0} & \frac{1}{\mu} \int_{0}^{\mu}\left\|A^{v} X B^{1-v}+A^{1-v} X B^{v}\right\| d v \\
& \leq \lim _{\mu \rightarrow 1} \frac{1}{1-\mu} \int_{\mu}^{1}\left\|A^{v} X B^{1-v}+A^{1-v} X B^{v}\right\| d v \\
& =\|A X+X B\| .
\end{aligned}
$$


Authors' contributions

YY carried out convex function. YF carried out unitarily invariant norm. GC carried out the calculation. All authors read and approved the final manuscript.

\section{Author details}

1School of Mathematics and Statistics, Southwest University, Chongqing, 400715, P.R. China. ${ }^{2}$ Department of

Mathematics and Information Engineering, Chongqing University of Education, Chongqing, 400067, P.R. China. ${ }^{3}$ Schoo

of Electronic Information Engineering, Southwest University, Chongqing, 400715, P.R. China.

\section{Acknowledgements}

This work is supported by NSF of China (Grant Nos. 11171364 and 11271301).

Received: 10 November 2013 Accepted: 8 January 2014 Published: 31 Jan 2014

\section{References}

1. Bhatia, R, Davis, C: More matrix forms of the arithmetic-geometric mean inequality. SIAM J. Matrix Anal. Appl. 14 132-136 (1993)

2. Feng, Y: Refinements of the Heinz inequalities. J. Inequal. Appl. 2012, 18 (2012). doi:10.1186/1029-242X-2012-18

3. Wang, S: Some new refinements of Heinz inequalities of matrices. J. Inequal. Appl. 2013, 424 (2013). doi:10.1186/1029-242X-2013-424

4. Kittaneh, F: On the convexity of the Heinz means. Integral Equ. Oper. Theory 68, 519-527 (2010)

5. Bullen, PS: A Dictionary of Inequalities. Pitman Monographs and Surveys in Pure and Applied Mathematics, vol. 97 Longman, Harlow (1998)

10.1186/1029-242X-2014-50

Cite this article as: Yan et al.: Refinements of the Heinz inequalities for matrices. Journal of Inequalities and Applications 2014, 2014:50

\section{Submit your manuscript to a SpringerOpen ${ }^{\circ}$ journal and benefit from:}

- Convenient online submission

- Rigorous peer review

- Immediate publication on acceptance

Open access: articles freely available online

- High visibility within the field

- Retaining the copyright to your article 